CTP TAMU-68/91

Imperial/TP/90-91/40

$\mathrm{IC} / 91 / 241$

September 1991

\title{
The $W_{3}$ String Spectrum
}

\author{
C.N. Pope,${ }^{1 \star}$ L.J. Romans,${ }^{2 \ddagger}$ E. Sezgin, ${ }^{1 \$}$ and K.S. Stelle ${ }^{3}$ \\ 1 Center for Theoretical Physics, Texas A\&M University, College Station \\ TX 77843-4242, USA \\ 2 Jet Propulsion Laboratory 301-150, California Institute of Technology, \\ Pasadena, CA 91109, USA \\ 3 The Blackett Laboratory, Imperial College, London SW7 $2 B Z$
}

\begin{abstract}
We study the spectrum of $W_{3}$ strings. In particular, we show that for appropriately chosen space-time signature, one of the scalar fields is singled out by the spin-3 constraint and is "frozen": no creation operators from it can appear in physical states and the corresponding momentum must assume a specific fixed value. The remaining theory is unitary and resembles an ordinary string theory in $d \neq 26$ with anomalies cancelled by appropriate background charges. In the case of the $W_{3}$ string, however, the spin-two "graviton" is massive.
\end{abstract}

* Supported in part by the U.S. Department of Energy, under grant DE-FG05-91ER40633.

$\mp$ Supported by a National Research Council-NASA/JPL Research Associateship.

\$ Supported in part by the National Science Foundation, under grant PHY-9106593. 


\section{Introduction}

The theory of $W_{3}$ strings starts from the construction of a non-chiral anomaly-free quantum $W_{3}$ gravity theory [1]. This is achieved [2] by taking a quantum realisation of the $W_{3}$ algebra with central charge $c=100$ [3]. Such realisations can be found for two [4] or more [5] scalar fields. In all cases, including that of $n=100$ scalar fields, these realisations require background charges for some of the scalar fields [5]. In the $W_{3}$ string theory, the $n$ scalar fields may be interpreted as spacetime coordinates. At the classical level, the equations of motion for the spin-2 and spin-3 gauge fields of $W_{3}$ gravity impose the vanishing of the left-moving and right-moving spin-2 and spin-3 matter currents. The realisations of the $W_{3}$ algebra found in [5] have the property that one scalar field, which we shall denote by $\varphi_{1}$, is distinguished in the way that it appears in the spin- 3 current; the other $n-1$ fields all appear only through their stress tensor. Upon use of the spin-2 constraints, this has the consequence of "freezing" the $\varphi_{1}$ coordinate [1]; i.e. the spin-3 constraints imply $\left(\partial \varphi_{1}\right)^{3}=\left(\bar{\partial} \varphi_{1}\right)^{3}=0$, and hence $\varphi_{1}$ is constant. In the present paper, we shall extend this classical picture of $W_{3}$ strings to the quantum level by discussing the quantum version of the "coordinate-freezing" phenomenon. We shall also discuss the spectrum of physical states. Although we shall mostly be considering closed $W_{3}$ strings, our discussion generalises straightforwardly to the case of open $W_{3}$ strings. Some preliminary quantum results have already been given in [1].

We shall denote the $n$ scalars by $\left(\varphi_{1}, \varphi_{2}, X^{\mu}\right)$, with $\mu=0,3,4, \ldots, n-1$. In order to have a Minkowski-signature spacetime, we shall take $X^{0}$ to be timelike and all the remaining coordinates to be spacelike.* The left-moving matter currents take the forms

$$
\begin{gathered}
T_{\text {mat }}=\mathcal{T}-\frac{1}{2}\left(\partial \varphi_{1}\right)^{2}-\frac{1}{2}\left(\partial \varphi_{2}\right)^{2}-\left(Q_{1} \partial^{2} \varphi_{1}+Q_{2} \partial^{2} \varphi_{2}\right) \\
W_{\text {mat }}=-\frac{2 \mathrm{i}}{\sqrt{261}}\left\{\frac{1}{3}\left(\partial \varphi_{1}\right)^{3}-\partial \varphi_{1}\left(\partial \varphi_{2}\right)^{2}+\left(Q_{1} \partial \varphi_{1} \partial^{2} \varphi_{1}-2 Q_{2} \partial \varphi_{1} \partial^{2} \varphi_{2}-Q_{1} \partial \varphi_{2} \partial^{2} \varphi_{2}\right)\right. \\
\left.\quad+\left(\frac{1}{3} Q_{1}^{2} \partial^{3} \varphi_{1}-Q_{1} Q_{2} \partial^{3} \varphi_{2}\right)+2 \partial \varphi_{1} \mathcal{T}+Q_{1} \partial \mathcal{T}\right\}
\end{gathered}
$$

where $\mathcal{T}$ is the stress tensor for the $D=n-2$ free scalar fields $X^{\mu}$ without background charges:

$$
\mathcal{T}=-\frac{1}{2} \eta_{\mu \nu} \partial X^{\mu} \partial X^{\nu}
$$

The right-moving currents $\widetilde{T}_{\text {mat }}$ and $\widetilde{W}_{\text {mat }}$ are defined similarly, with $\partial$ replaced by $\bar{\partial}$. The background charges $Q_{1}$ and $Q_{2}$ for $\varphi_{1}$ and $\varphi_{2}$ are given by [2]

$$
\begin{aligned}
& Q_{1}^{2}=\frac{49}{8} \\
& Q_{2}^{2}=\frac{1}{12}\left(\frac{49}{2}-D\right),
\end{aligned}
$$

* Note that we have made a different choice of signature for the $\varphi_{1}$ coordinate from that in [1]. This is in order to ensure the unitarity of the theory, as we shall see later. 
and are needed to obtain a total matter central charge $c_{\text {mat }}=100$. Initially, we shall assume that $D \leq 24$, so that $Q_{2}$ is real.

We expand the coordinates in Laurent series as usual:

$$
\begin{aligned}
\mathrm{i} \partial \varphi_{1} & =\sum_{m} \alpha_{m}^{1} z^{-m-1} \\
\mathrm{i} \partial \varphi_{2} & =\sum_{m} \alpha_{m}^{2} z^{-m-1} \\
\mathrm{i} \partial X^{\mu} & =\sum_{m} \alpha_{m}^{\mu} z^{-m-1} .
\end{aligned}
$$

Upon quantisation, the oscillators $\alpha_{m}$ satisfy the commutation relations

$$
\left[\alpha_{m}^{i}, \alpha_{n}^{j}\right]=m \eta^{i j} \delta_{m+n, 0} \quad i=1,2, \mu
$$

where $\eta^{00}=-1$ and all the other diagonal elements equal +1 . Expanding the spin- 2 current $T_{\text {mat }}=\sum_{n} L_{n} z^{-n-2}$ and the spin-3 current $W_{\text {mat }}=\sum_{n} W_{n} z^{-n-3}$ in terms of the oscillators, we have

$$
\begin{aligned}
& L_{n}=\frac{1}{2} \sum_{m}:\left(\alpha_{n-m}^{1} \alpha_{m}^{1}+\alpha_{n-m}^{2} \alpha_{m}^{2}+\eta_{\mu \nu} \alpha_{n-m}^{\mu} \alpha_{m}^{\nu}\right):-\mathrm{i}(n+1)\left(Q_{1} \alpha_{n}^{1}+Q_{2} \alpha_{n}^{2}\right) \\
& W_{n}=\frac{2}{\sqrt{261}}\left\{\sum_{p, q}:\left(\frac{1}{3} \alpha_{n-p-q}^{1} \alpha_{p}^{1} \alpha_{q}^{1}-\alpha_{n-p-q}^{1} \alpha_{p}^{2} \alpha_{q}^{2}-\alpha_{n-p-q}^{1} \alpha_{p}^{\mu} \alpha_{q}^{\nu} \eta_{\mu \nu}\right):\right. \\
& \quad+\mathrm{i} \sum_{m}(m+1):\left(-Q_{1} \alpha_{n-m}^{1} \alpha_{m}^{1}+2 Q_{2} \alpha_{n-m}^{1} \alpha_{m}^{2}+Q_{1} \alpha_{n-m}^{2} \alpha_{m}^{2}+Q_{1} \alpha_{n-m}^{\mu} \alpha_{m}^{\nu} \eta_{\mu \nu}\right): \\
& \left.+(n+1)(n+2)\left(-\frac{1}{3} Q_{1}^{2} \alpha_{n}^{1}+Q_{1} Q_{2} \alpha_{n}^{2}\right)\right\}
\end{aligned}
$$

The hermiticity conditions $L_{n}^{\dagger}=L_{-n}$ and $W_{n}^{\dagger}=W_{-n}$ imply that $\alpha_{n}^{i \dagger}=\alpha_{-n}^{i},(i=1,2, \mu)$, except for $\alpha_{0}^{1}$ and $\alpha_{0}^{2}$, which must satisfy conditions that are modified by the background charges:

$$
\alpha_{0}^{1 \dagger}=\alpha_{0}^{1}-2 \mathrm{i} Q_{1}, \quad \alpha_{0}^{2 \dagger}=\alpha_{0}^{2}-2 \mathrm{i} Q_{2}
$$

For these, it is convenient to introduce shifted oscillators $\hat{\alpha}_{0}^{1}$ and $\hat{\alpha}_{0}^{2}$ that are Hermitean:

$$
\hat{\alpha}_{0}^{1}=\alpha_{0}^{1}-\mathrm{i} Q_{1}, \quad \hat{\alpha}_{0}^{2}=\alpha_{0}^{2}-\mathrm{i} Q_{1}
$$

As in ordinary string theory, the quantum constraints that are to be imposed on physical states are those corresponding to the Laurent-mode expansion coefficients for the currents with non-negative indices. It is sufficient to impose just the constraints for $L_{0}, L_{1}, L_{2}$ and $W_{0}$ (together with their right-moving counterparts), since all the rest follow by commutation. It is known from the BRST-nilpotency conditions derived in [3] that the intercepts for $L_{0}$ 
and $W_{0}$ are -4 and 0 respectively, so the constraints to be imposed on physical states are $[1]$ :

$$
\begin{array}{llll}
L_{0}-4=0, & L_{1}=0, & L_{2}=0, & W_{0}=0 \\
\widetilde{L}_{0}-4=0, & \widetilde{L}_{1}=0, & \widetilde{L}_{2}=0, & \widetilde{W}_{0}=0
\end{array}
$$

It is convenient to make use of the $L_{n}$ constraints to simplify $W_{0}$. After imposing the $L_{n}$ constraints in $(10 a)$, we find that $W_{0}$ can be rewritten as

$$
\begin{aligned}
W_{0}=\frac{1}{3 \sqrt{261}} & \left\{5 \sum^{\prime}: \alpha_{-p-q}^{1} \alpha_{p}^{1} \alpha_{q}^{1}:+\hat{\alpha}_{0}^{1}\left(8\left(\hat{\alpha}_{0}^{1}\right)^{2}+36 \mathcal{N}^{(1)}+1\right)\right. \\
& \left.-12 \mathrm{i} Q_{1} \sum_{n>0} n \alpha_{-n}^{1} \alpha_{n}^{1}-12 \sum_{n>0} \hat{L}_{-n} \alpha_{n}^{1}\right\}
\end{aligned}
$$

where $\hat{\alpha}_{0}^{1}$ is given by $(9), \mathcal{N}^{(1)}$ denotes the number operator for $\alpha^{1}$ oscillators

$$
\mathcal{N}^{(1)}=\sum_{n>0} \alpha_{-n}^{1} \alpha_{n}^{1}
$$

and $\hat{L}_{n}$ denotes the terms in $L_{n}$ that do not involve the $\alpha_{m}^{1}$ oscillators,

$$
\hat{L}_{n}=\frac{1}{2} \sum_{m}:\left(\alpha_{n-m}^{2} \alpha_{m}^{2}+\eta_{\mu \nu} \alpha_{n-m}^{\mu} \alpha_{m}^{\nu}\right):-\mathrm{i}(n+1) Q_{2} \alpha_{n}^{2}
$$

The prime on the summation symbol in the first term in (11) indicates that only terms with all Laurent indices non-zero are included.

To construct the physical states, we begin by defining the Fock vacuum $|v a c\rangle$ :

$$
\alpha_{n}^{i}|\operatorname{vac}\rangle=0, \quad i=1,2, \mu ; \quad n \geq 0
$$

The "tachyon" state $|p\rangle$ is given by

$$
|p\rangle=\exp \left(\mathrm{i} p_{1} \varphi^{1}(0)+\mathrm{i} p_{2} \varphi^{2}(0)+\mathrm{i} p_{\mu} X^{\mu}(0)\right)|\operatorname{vac}\rangle
$$

and higher states are built by acting on (15) with linear combinations of products of the creation operators $\alpha_{n}^{i}, n<0$. Of course, as usual in closed string theory, there is a global constraint that the left-moving and right-moving level numbers must be equal. With this understood, we shall concentrate in the following on just the left-moving sector. 


\section{Freezing the $\varphi^{1}$ coordinate}

We now argue that no physical states can involve any $\alpha_{n}^{1}$ creation operators. This is the quantum version of the "coordinate-freezing" phenomenon mentioned above at the classical level [1]. Consider imposing the $W_{0}$ constraint, with $W_{0}$ given by (11), on a general level- $N$ state in the theory. (The level $N$ is the sum of the Laurent-mode indices in each monomial of creation operators forming the state.) Amongst the monomials at level $N$ will be ones that involve only $\alpha^{1}$ oscillators. From the form of (11), we see that all terms except the last one preserve the $\alpha^{1}$ level number $N^{(1)}$, whilst the last term lowers it. After applying $W_{0}$ to the state, we may focus on the terms that still have $\alpha^{1}$ level $N^{(1)}=N$. (The final term in (11) may thus be neglected in this discussion.) The coefficient of each such independent monomial must vanish, by virtue of the $W_{0}=0$ constraint. This gives as many homogeneous equations as there are coefficients to be determined. As we shall see in examples below, these systems are non-degenerate, and hence the coefficients of all the monomials with $\alpha^{1}$ level equal to $N$ must vanish. The argument can now be repeated at level $N^{(1)}=N-1$ in $\alpha^{1}$ oscillators, so that we have just one $\alpha_{1}^{j \neq 1}$ oscillator in each monomial. Again, we focus on terms of highest $\alpha^{1}$ level, now equal to $N-1$, after applying $W_{0}$. (As before, the last term in (11) may be neglected.) The $\alpha_{1}^{j \neq 1}$ oscillators commute with the relevant terms in (11), and again we obtain a non-degenerate set of homogeneous equations for the monomial coefficients. Proceeding iteratively, we find that all monomials containing $\alpha^{1}$ creation operators must have zero coefficients.

Let us illustrate the above discussion with some examples. At level 1, the only term involving $\alpha^{1}$ is of the form

$$
\lambda \alpha_{-1}^{1}|p\rangle .
$$

(Since we shall focus on the terms involving a surviving $\alpha_{-1}^{1}$ after applying $W_{0}$, and since no terms in $W_{0}$ can increase the $\alpha^{1}$ level $N^{(1)}$, we do not need to consider the other possible monomials in a level- 1 state.) Thus applying $W_{0}$, we find that the coefficient of $\alpha_{-1}^{1}$ is proportional to

$$
\lambda\left(\hat{p}_{1}\left(8\left(\hat{p}_{1}\right)^{2}+37\right)-12 \mathrm{i} Q_{1}\right),
$$

where $\hat{p}_{1}$ is the eigenvalue of the Hermitean operator $\hat{\alpha}_{0}^{1}$ defined in (9). Since $\hat{p}_{1}$ is real, it follows from the $W_{0}$ constraint that $\lambda$ is zero. Thus, at level 1 there can be no $\alpha^{1}$ creation operators in physical states.

At level 2, the monomials with $\alpha^{1}$ level 2 have the form

$$
\left(\lambda \alpha_{-2}^{1}+\mu \alpha_{-1}^{1} \alpha_{-1}^{1}\right)|p\rangle .
$$

After applying $W_{0}$, the coefficients of the monomials $\alpha_{-2}^{1}$ and $\alpha_{-1}^{1} \alpha_{-1}^{1}$ must independently vanish. This gives the set of equations

$$
\left(\begin{array}{cc}
F_{2}-48 \mathrm{i} Q_{1} & 30 \\
30 & F_{2}-24 \mathrm{i} Q_{1}
\end{array}\right)\left(\begin{array}{l}
\lambda \\
\mu
\end{array}\right)=0,
$$


where $F_{N^{(1)}}$ is the real quantity

$$
F_{N^{(1)}}=\hat{p}_{1}\left(8\left(\hat{p}_{1}\right)^{2}+36 N^{(1)}+1\right) .
$$

The condition for (19) to admit non-zero solutions for $\lambda$ and $\mu$ (i.e. the condition that the determinant of the matrix of coefficients vanish) implies that

$$
F_{2}=\left(36 \mathrm{i} \pm \frac{12}{7}\right) Q_{1}
$$

which is impossible since $F_{2}$ is real. Thus $\lambda$ and $\mu$ must be zero. This shows that at level 2 , physical states can be at most of level 1 in $\alpha^{1}$ oscillators. But our previous discussion for level 1 states now carries over without modification to show that the coefficients of these monomials must also be zero. Therefore no $\alpha^{1}$ creation operators can appear in level-2 physical states.

At higher levels the above pattern repeats. We have explicitly checked up to level 5 that the $W_{0}$ constraint produces a non-degenerate set of homogeneous equations for the coefficients of all the possible monomials involving $\alpha^{1}$ oscillators, showing that at least up to this level, no $\alpha^{1}$ creation operators can occur in physical states. The reason is always that the roots of the various vanishing-determinant conditions would require complex solutions for the real quantities $F_{N^{(1)}}$. The pattern of the imaginary parts becomes clear from our sequence of low-level examples. The imaginary parts $y_{r}, r=1, \ldots, P_{N^{(1)}}$ of the roots (where $P_{N^{(1)}}$ is the number of possible $\alpha^{1}$ monomials at level $\left.N^{(1)}\right)$ turn out in all cases to be given by the formula

$$
\left.y_{r}=\frac{1}{2}\left(u_{r}+u_{\left(P_{N}(1)\right.}+1-r\right)\right), \quad r=1, \ldots, P_{N^{(1)}} .
$$

The $u_{r}$ in (22) are the imaginary parts of the diagonal entries in the matrix of equation coefficients, organised in monotonically decreasing value. Thus, $u_{r}$ is the eigenvalue of the diagonal operator $12 Q_{1} \sum_{n>0} n \alpha_{-n}^{1} \alpha_{n}^{1}$ corresponding to the eigenvector created by the $r^{\prime}$ th monomial. Since all the $u_{r}$ are positive, it follows that the $y_{r}$ are all non-zero. Consequently, the homogeneous equations for the $\alpha^{1}$ monomial coefficients are non-degenerate and so all these coefficients must vanish.

As a final illustration of these ideas, we give the $7 \times 7$ matrix of equation coefficients for the level-5 monomials $\alpha_{-5}^{1}, \alpha_{-4}^{1} \alpha_{-1}^{1}, \alpha_{-3}^{1} \alpha_{-2}^{1}, \alpha_{-3}^{1}\left(\alpha_{-1}^{1}\right)^{2},\left(\alpha_{-2}^{1}\right)^{2} \alpha_{-1}^{1}, \alpha_{-2}^{1}\left(\alpha_{-1}^{1}\right)^{3}$ and $\left(\alpha_{-1}^{1}\right)^{5}$ :

$\left(\begin{array}{ccccccc}F_{5}-300 \mathrm{i} Q_{1} & 120 & 180 & 0 & 0 & 0 & 0 \\ 150 & F_{5}-204 \mathrm{i} Q_{1} & 0 & 180 & 120 & 0 & 0 \\ 150 & 0 & F_{5}-156 \mathrm{i} Q_{1} & 30 & 120 & 0 & 0 \\ 0 & 120 & 30 & F_{5}-132 \mathrm{i} Q_{1} & 0 & 180 & 0 \\ 0 & 60 & 90 & 0 & F_{5}-108 \mathrm{i} Q_{1} & 90 & 0 \\ 0 & 0 & 0 & 90 & 60 & F_{5}-84 \mathrm{i} Q_{1} & 300 \\ 0 & 0 & 0 & 0 & 0 & 30 & F_{5}-60 \mathrm{i} Q_{1}\end{array}\right)$


It is straightforward to show that the characteristic equation gives roots whose imaginary parts are in accordance with (22),

$$
F_{5}=132 \mathrm{i} Q_{1},\left(180 \mathrm{i} \pm \frac{120}{7}\right) Q_{1},\left(144 \mathrm{i} \pm \frac{60}{7}\right) Q_{1},\left(132 \mathrm{i} \pm \frac{24}{7}\right) Q_{1}
$$

Thus, there are no real solutions for $F_{5}$.

Having seen that the physical states involve no $\alpha^{1}$ oscillators, the remaining content of the $W_{0}$ constraint on physical states is, from (11),

$$
\hat{p}_{1}\left(8\left(\hat{p}_{1}\right)^{2}+1\right)=0
$$

Thus, for all physical states $\hat{p}_{1}$ is frozen:

$$
\hat{p}_{1}=0
$$

\section{The construction of physical states}

We have seen from the discussion in the previous section that the $W_{0}$ constraint simply has the effect of eliminating the $\varphi^{1}$ coordinate from the theory, and freezing the $\hat{p}_{1}$ momentum as in (26). In terms of the original unshifted momentum component $p_{1}$ (the eigenvalue of the non-hermitean operator $\alpha_{0}^{1}$ ), this implies that it simply takes the fixed imaginary value $p_{1}=\mathrm{i} Q_{1}$. The $W_{n}$ constraints then play no further rôle in the theory. The construction of physical states now closely parallels that for string theory with a background charge.

Although we have chosen the background charge $Q_{2}$ to lie in the $\varphi_{2}$ direction, we could in fact equally well allow it to lie in some unspecified direction in the $(D+1)$-dimensional spacetime spanned by the coordinates $\varphi_{2}$ and $X^{\mu}$, as long as the (vector) background-charge parameter $Q^{a}, a=2, \mu$, has the same magnitude as $Q_{2}$, i.e.

$$
Q^{a} Q_{a}=\frac{1}{12}\left(\frac{49}{2}-D\right)
$$

It is sometimes advantageous to do this, since this enables us to write the constraints and physical states in a formally $(D+1)$-dimensional Lorentz-covariant fashion. Note, however, that since the constant vector $Q^{a}$ singles out a direction in spacetime, $(D+1)$-dimensional Lorentz invariance is broken. Thus, bearing in mind that physical states do not involve $\alpha^{1}$ creation operators, we may rewrite the $L_{n}$ constraint operators, for application onto such states, as

$$
\begin{aligned}
L_{0}-4 & =\frac{1}{2} \hat{\alpha}_{0}^{a} \hat{\alpha}_{0}^{b} \eta_{a b}+\mathcal{N}-4+\frac{1}{2} Q_{1}^{2}+\frac{1}{2} Q^{a} Q_{a}, \\
& =\frac{1}{2} \hat{\alpha}_{0}^{a} \hat{\alpha}_{0}^{b} \eta_{a b}+\mathcal{N}+\frac{1}{12}(2-D), \\
L_{n} & =\left(\hat{\alpha}_{0 a}-i n Q_{a}\right) \alpha_{n}^{a}+\frac{1}{2} \eta_{a b} \sum^{\prime}: \alpha_{n-m}^{a} \alpha_{m}^{b}:, \quad n>0 .
\end{aligned}
$$


The (hermitean) operators $\hat{\alpha}_{0}^{a}$ are related to $\alpha_{0}^{a}$ by

$$
\hat{\alpha}_{0}^{a}=\alpha_{0}^{a}-\mathrm{i} Q^{a}
$$

$\mathcal{N}$ is the number operator for the $\alpha^{a}$ oscillators,

$$
\mathcal{N}=\sum_{m>0} \eta_{a b} \alpha_{-m}^{a} \alpha_{m}^{b}
$$

and the prime on the summation in (28b) indicates that only terms with non-zero Laurent indices are to be included. Note that the original momentum components $p^{a}$ have a fixed imaginary part $Q^{a}$.

Physical states are built up from the Fock vacuum by applying linear combinations of monomials in the creation operators $\alpha^{a}$ that are homogeneous in the overall level number $N$ (the eigenvalue of $\mathcal{N}$ ). As we remarked earlier, in closed-string theory one has a global constraint that the left-moving and right-moving level numbers $N$ and $\widetilde{N}$ must be equal. It is to be understood in what follows that we are focussing on just the left-moving sector of such physical states.

In this section, we shall build the first few levels of physical states. The lowest-lying state, at level $N=0$, is the "tachyon," given by (15) with $p_{1}=\mathrm{i} Q_{1}$. The only remaining non-trivial constraint in this case is the mass-shell condition $\left(L_{0}-4\right)|p\rangle=0$, where $L_{0}-4$ is given by $(28 a)$. Thus we have*

$$
-\hat{p}^{a} \hat{p}_{a}=\frac{1}{12}(2-D)
$$

We shall postpone discussing the issue of masslessness until the next section. For now, we just remind the reader that since there is a background charge in the $(D+1)$-dimensional spacetime, which breaks the $(D+1)$-dimensional Lorentz covariance, the notion of mass is modified. The Fock vacuum, by definition, will be taken to have positive norm. This implies that the tachyon will also have positive norm. Note that, because of the presence of the background charge, the inner product for tachyon states reads

$$
\begin{aligned}
\left\langle p^{\prime} \mid p\right\rangle & =\delta\left(p^{\prime}+p-2 \mathrm{i} Q\right) \\
& =\delta\left(\bar{p}^{\prime}+p\right) .
\end{aligned}
$$

At the next level, $N=\widetilde{N}=1$, the left-moving sector of a physical state takes the form

$$
|\xi, p\rangle=\xi_{a} \alpha_{-1}^{a}|p\rangle
$$

* Similar discussions have been given in [6] for the case of a string in $d>26$ dimensions with a background charge in the time direction. 
The $L_{0}$ and $L_{1}$ constraints are non-trivial, giving, respectively,

$$
-\hat{p}^{a} \hat{p}_{a}=\frac{1}{12}(26-D)
$$

and

$$
\left(\hat{p}^{a}-\mathrm{i} Q^{a}\right) \xi_{a}=0 .
$$

Since $\hat{p}^{a}$ is the eigenvalue of the hermitean operator $\hat{\alpha}_{0}^{a}$ given in (29), (35) may be written as

$$
\bar{p}^{a} \xi_{a}=0 .
$$

The norm of the state (33) is a positive number times

$$
S \equiv \eta^{a b} \bar{\xi}_{a} \xi_{b} .
$$

We may use (36) to solve for the timelike component $\xi_{0}$, and rewrite (37) as

$$
S=\bar{\xi}_{A} \xi_{B} M^{A B} \equiv \bar{\xi}_{A} \xi_{B}\left(\delta^{A B}-\frac{p^{A} \bar{p}^{B}}{\left|p^{0}\right|^{2}}\right),
$$

where the indices $A, B \ldots$ run over the spatial directions $2,3, \ldots, D+1$. Clearly, the matrix $M^{A B}$ has $D$ unit eigenvalues (corresponding to the $D$ eigenvectors $V_{A}$ satisfying $\bar{p}^{A} V_{A}=0$ ), and one special eigenvalue, which corresponds to the eigenvector $p_{A}$. On using the mass-shell condition (34), which can be rewritten using (27) and (29) as $-\bar{p}^{a} p_{a}=\frac{1}{8}$, we see that this eigenvalue is equal to

$$
\frac{1}{8\left|p_{0}\right|^{2}} \text {. }
$$

Thus all the states in (33) have positive norm. This contrasts with the situation in ordinary string theory, for which the special eigenvalue analogous to (39) would be zero. We shall discuss this further in the next section.

At level 2 in the left-moving sector, physical states will have the form

$$
|\beta, \epsilon, p\rangle=\left(\beta_{a b} \alpha_{-1}^{a} \alpha_{-1}^{b}+\epsilon_{a} \alpha_{-2}^{a}\right)|p\rangle .
$$

The conditions following from the $L_{0}, L_{1}$ and $L_{2}$ constraints at this level are

$$
\begin{aligned}
-\hat{p}^{a} \hat{p}_{a} & =\frac{1}{12}(50-D), \\
\beta_{a b}\left(\hat{p}^{b}-\mathrm{i} Q^{b}\right)+\epsilon_{a} & =0, \\
2\left(\hat{p}^{a}-2 \mathrm{i} Q^{a}\right) \epsilon_{a}+\beta_{a}{ }^{a} & =0 .
\end{aligned}
$$

Using (27) and (29), these may be rewritten as

$$
\begin{aligned}
-\bar{p}^{a} p_{a} & =\frac{17}{8}, \\
\beta_{a b} \bar{p}^{b}+\epsilon_{a} & =0, \\
2\left(\bar{p}^{a}-\mathrm{i} Q^{a}\right) \epsilon_{a}+\beta_{a}{ }^{a} & =0 .
\end{aligned}
$$


We can use (42b) to solve for $\epsilon_{a}$, giving $\epsilon_{a}=-\beta_{a b} \bar{p}^{b}$. From (42c), $\beta_{a b}$ must then satisfy the constraint $\beta_{a}{ }^{a}=2 \bar{p}^{a}\left(\bar{p}^{b}-\mathrm{i} Q^{b}\right) \beta_{a b}$.

It is useful to decompose $\beta_{a b}$ in the form

$$
\beta_{a b}=\lambda \eta_{a b}+\mu p_{a} p_{b}+\beta_{(a}^{\mathrm{T}} p_{b)}+\beta_{a b}^{\mathrm{TT}}
$$

where

$$
\bar{p}^{a} \beta_{a}^{\mathrm{T}}=0, \quad \bar{p}^{a} \beta_{a b}^{\mathrm{TT}}=0, \quad \beta_{a}^{\mathrm{TT} a}=0 .
$$

Substituting this into the constraint on $\beta_{a b}$ derived from $(42 b, c)$, one finds that

$$
\frac{\mathrm{i}}{8} \beta_{a}^{\mathrm{T}} Q^{a}=\left(2 \lambda-\frac{3}{4} \mu\right)\left(\frac{119}{8}+3 \mathrm{i} p_{a} Q^{a}\right) .
$$

Using this, and inserting (42b) and (43) into the expression for the norm, which is a positive multiple of $S \equiv \bar{\epsilon}_{a} \epsilon^{a}+\bar{\beta}_{a b} \beta^{a b}$, gives

$$
S=\left(\frac{17}{8}\right)^{2}\left[-8|\lambda|^{2}-\frac{9}{8}|\mu|^{2}+3 \lambda \bar{\mu}+3 \bar{\lambda} \mu\right]+\frac{17}{256} \bar{\beta}_{a}^{\mathrm{T}} \beta^{\mathrm{T} a}+\bar{\beta}_{a b}^{\mathrm{TT}} \beta^{\mathrm{TT} a b} .
$$

The constraint (45) implies that $\beta_{a}^{\mathrm{T}}$ must be non-zero when $\mu \neq \frac{8}{3} \lambda$, so the quadratic form (46) still has a mixing between the $\lambda, \mu$ and $\beta_{a}^{\mathrm{T}}$ modes. In fact, it is just the single mode in $\beta_{a}^{\mathrm{T}}$ that is parallel to $Q_{a}$ that mixes with $\lambda$ and $\mu$. Thus we must diagonalise the corresponding three-dimensional quadratic form. We do this by noting that we may, without loss of generality, choose $Q_{a}$ to lie in the $\varphi_{2}$ direction, and furthermore we may use the remaining $(D-1)$-dimensional rotation group for the coordinates $\left(X^{3}, \ldots, X^{D+1}\right)$ to choose $p_{a}$ such that only $p_{0}, p_{2}$ and $p_{3}$ are non-zero. Solving for $\beta_{0}^{\mathrm{T}}$ from (44), and for $\beta_{2}^{\mathrm{T}}$ from (45), we obtain the three-dimensional contribution $S_{3}$ to $S$, sesquilinear in $\left(\lambda, \mu, \beta_{3}^{\mathrm{T}}\right)$ :

$$
\begin{aligned}
S_{3} & =Q_{2}^{-2}\left(\frac{17}{8}+p_{3}^{2}\right)|6 \mu-16 \lambda|^{2}\left[\left(\frac{119}{8}\right)^{2}+9 Q_{2}^{2}\left|p_{2}\right|^{2}-\frac{357}{4} Q^{2}\right] \\
& +\left\{\mathrm{i} Q_{2}^{-1} \beta_{3}^{\mathrm{T}}(6 \bar{\mu}-16 \bar{\lambda})\left(\frac{119}{8}-3 \mathrm{i} \bar{p}_{2} Q_{2}\right) p_{2} p_{3}+\text { c.c. }\right\} \\
& +\left(\frac{17}{8}+\left|p_{2}\right|^{2}\right)\left|\beta_{3}^{\mathrm{T}}\right|^{2}-17 p_{0}^{2}\left(32|\lambda|^{2}+\frac{9}{2}|\mu|^{2}-12 \lambda \bar{\mu}-12 \bar{\lambda} \mu\right) .
\end{aligned}
$$

(Note that since $Q_{a}$ has been chosen to lie along the 2 direction, the momentum components $p_{a}$ in all other directions are real.) It is straightforward to see that the quadratic form (47) has eigenvalues $\sigma_{1}, \sigma_{2}$ and $\sigma_{3}$ satisfying

$$
\begin{aligned}
& \sigma_{1}=0 \\
& \sigma_{2} \sigma_{3}=\frac{1241}{128 Q_{2}^{2}} p_{0}^{2}\left(14161-5848 Q_{2}^{2}+512 Q_{2}^{4}+512 Q_{2}^{2} \hat{p}_{2}^{2}\right) \\
& \sigma_{2}+\sigma_{3}= \frac{1}{128 Q_{2}^{2}}\left(17573801-7257096 Q_{2}^{2}+635520 Q_{2}^{4}+8270024 p_{3}^{2}-3415232 Q_{2}^{2} p_{3}^{2}\right. \\
&\left.+336384 Q_{2}^{4} p_{3}^{2}+635520 Q_{2}^{2} \hat{p}_{2}^{2}+336384 Q_{2}^{2} p_{3}^{2} \hat{p}_{2}^{2}\right) .
\end{aligned}
$$


From (3), we see that $Q_{2}^{2}$ must lie in the range $\frac{1}{24} \leq Q_{2}^{2} \leq \frac{49}{24}$, and it is easy to verify that the expressions $(48 b)$ and $(48 c)$ are always positive for $Q_{2}^{2}$ in this range. Thus $S_{3}$ represents the contribution of one zero-norm state and two positive-norm states in the level-2 physical spectrum.

To analyse the remaining states, one may use the expressions in (44) to solve for the components of $\beta_{a}^{\mathrm{T}}$ and $\beta_{a b}^{\mathrm{TT}}$ with timelike indices in terms of $\beta_{A}^{\mathrm{T}}$ and $\beta_{A B}^{\mathrm{TT}}$. The eigenvalues of the resulting quadratic form may then be evaluated in a similar fashion to our level-1 calculation. One finds that the $\bar{\beta}_{a}^{\mathrm{T}} \beta^{\mathrm{T} a}$ and $\bar{\beta}_{a b}^{\mathrm{TT}} \beta^{\mathrm{TT} a b}$ terms in (46) have positive-definite norm. It should be emphasised that our results for the norms of these states are not sensitive to the value of $D$ (except that up until now we have assumed that $D \leq 24$ in order for $Q_{2}$ to be real). This dimension independence arises because the background charges ensure that we have a critical theory.

The restriction $D \leq 24$ that we have imposed up until now in order to keep $Q_{a}$ real may simply be relaxed by analytically continuing our results now to general $D$. All the above norm calculations give $D$-independent results and remain unchanged. For $D \geq 25$, the analogue of the inner product relation (32) is

$$
\left\langle p^{\prime} \mid p\right\rangle=\delta\left(p^{\prime}+p-2 B\right)
$$

where $B_{a}=\mathrm{i} Q_{a}$ is real. Thus, momentum conservation requires the same sort of shifts in $p^{a}$ in norm calculations as those that were obtained above in the $D \leq 24$ region under complex conjugation $p_{a} \rightarrow \bar{p}_{a}=p_{a}-2 \mathrm{i} Q_{a}$. Consequently, our above results extend straightforwardly to the $D \geq 25$ region.

\section{Masses and compactification}

We have seen above that the level-1 states of the $W_{3}$ string differ from those of the ordinary bosonic string in that there is no zero-norm state. This is an indication that the physical states at this level are not massless. What then is one to make of the mass-shell condition (34), which seems to indicate the possibility of massless states? (This question has already been raised in [1].) Since the presence of the background charge (27) breaks Lorentz covariance in $D+1$ dimensions, there is not a clear meaning to "mass" as a Lorentz Casimir operator. In order to clarify this issue, we shall now consider compactifying the dimension associated with the background charge and shall discuss the resulting Lorentz-covariant mass formula in the remaining $D$ dimensions. For these purposes, it is convenient to revert to having a definite choice of the direction associated to the background charge and we shall again let this be the $\varphi_{2}$ direction.

In order to compactify $\varphi_{2}$, i.e. to let it take its values in a circle $S^{1}$, we have to ensure that there is a symmetry of the quantum path integral under some shift $\Delta$ in the value of $\varphi_{2}$ so that we can make the identification of $\varphi_{2}$ with $\varphi_{2}+\Delta$. Moreover, this shift symmetry 
has to hold at any order in the string loop expansion and with any number of external states ("punctures"). String loop calculations are done with an Euclidean worldsheet, so the path integral integrand is $\exp \left(-\pi^{-1} \int \mathcal{L}\right)$. The field $\varphi_{2}$ enters the Lagrangian $\mathcal{L}$ as $\mathcal{L}_{2}=-\frac{1}{2} \bar{\partial} \varphi_{2} \partial \varphi_{2}-h T_{2}$ (plus the $\widetilde{T}_{2}, W$ and $\widetilde{W}$ current terms, with which we are not concerned at this point), where

$$
\mathcal{T}_{2}=-\frac{1}{2}\left(\partial \varphi_{2}\right)^{2}-Q_{2} \partial^{2} \varphi_{2}
$$

By integrating the derivatives in the background charge term over onto the spin-2 gauge field $h$, and noting that in light-cone gauge the Ricci scalar $R$ is given by $R=2 \partial^{2} h$, we see that the background charge term in (50) may be reinterpreted [6] as a dilaton coupling $\mathcal{L}_{\text {dil }}=R \Phi$ with $\Phi=\frac{1}{2} Q_{2} \varphi_{2}$. Thus, we have to ensure that the shift term $\exp \left(-(2 \pi)^{-1} Q_{2} \Delta \int R\right)$ in the path integral is equal to unity. The quantity $(4 \pi)^{-1} \int R=\chi$ is the worldsheet Euler number and so takes integer values. (For unpunctured worldsheets, $\chi$ takes even values, but punctures can make it odd.) We must also have $Q_{2}=-\mathrm{i} B_{2}$ imaginary (so we require $D \geq 25$ here). Thus, for compactification of the $\varphi_{2}$ direction, there will be a symmetry under shifts of $\varphi_{2}$ by $\Delta=\pi / B_{2}$.

The above discussion shows that we may compactify the $\varphi_{2}$ direction on a circle of circumference $\pi / B_{2}$ in the case $D \geq 25$. Under these circumstances, the momentum $p_{2}$ can take the values

$$
p_{2}=2 n B_{2}, \quad n \in \mathbb{Z} .
$$

This implies that the $D$-dimensional mass formula following from (34) upon compactification becomes

$$
\mathcal{M}^{2}=-p_{\mu} p^{\mu}=\frac{1}{12}(26-D)+\frac{1}{12}(2 n-1)^{2}\left(D-\frac{49}{2}\right) .
$$

From this it is clear that there are no massless states at this level in the compactified theory: the allowed values of $p_{2}$ cause $\mathcal{M}^{2}$ to "jump over" the value zero. This accords with what we found in our norm calculations in section 3, where there was no longitudinal null state at level 1. Since this is the level that, when combined with level 1 in the right-moving sector, gives the graviton in ordinary string theory, we come to the conclusion that $W_{3}$ strings do not contain a massless graviton. It seems likely that the impossibility of massless states will continue at higher levels as well. This conclusion is in contrast to the general arguments given in [7], which suggested that there should be higher-spin massless states. The arguments of [7] were based upon the observation that the intercept for the $L_{0}$ mass-shell constraint is $(-4)$, rather than the $(-1)$ of ordinary string theory, thus suggesting a downward shift of the $W_{3}$ (mass) $)^{2}$ eigenvalues relative to ordinary string theory. However, this observation overlooks the fact that $W_{3}$ strings require background charges, which more than overcome the negative displacement of the intercept. In fact, as we have seen, the relative downward shift of -3 in the $L_{0}$ intercept is counteracted by an upward shift of $\frac{1}{2} Q_{1}^{2}=3+\frac{1}{16}$ from the fixed background charge in the $\varphi_{1}$ direction. 
For the level-1 states, the norm calculation in section 3 led to the conclusion, supported by our above discussion for the compactified theory at $D \geq 25$, that it is misleading to view the left-hand side of the mass-shell condition (34) as the "(mass) ${ }^{2}$ operator." In fact, it would seem that a better indication of mass is provided by the quantity $-\bar{p}^{a} p_{a}$, which, for level-1 takes the value

$$
-\bar{p}^{a} p_{a}=\frac{1}{8}
$$

independent of $D$. A similar calculation at level-1 in ordinary string theory, with criticality achieved by taking $D$ spacetime coordinates with an appropriate background charge, would yield the results $-\hat{p}^{a} \hat{p}_{a}=\frac{1}{12}(26-D)$, and $-\bar{p}^{a} p_{a}=0$. Again, it would seem that $-\bar{p}^{a} p_{a}$ provides the better measure of mass: In this case one finds by calculating the norm of the level-1 states that for any $D$ there is always a null state, corresponding to a gauge degree of freedom, and so it seems appropriate to view the level-1 states of ordinary critical string theory as comprising a massless vector for all $D$.

If one chooses a value for $D$ that is $\leq 24$, then the compactification procedure that we have just discussed cannot be implemented, since the background charge $Q_{2}$ is then real and so the integrand of the functional integral will not exhibit any periodicity under shifts in $\varphi_{2}$. Thus, under these circumstances one cannot use compactification to circumvent the difficulties of defining mass in the $(D+1)$-dimensional spacetime with background charge. However, having seen in the case of the spin-1 states that the most appropriate mass operator appears to be $-\bar{p}^{a} p_{a}$ rather than $-\hat{p}^{a} \hat{p}_{a}$, one might argue that the former should also be regarded as defining the mass for states at all levels. In particular, according to this viewpoint, the mass for the "tachyon" state (15) (with $p_{1}=\mathrm{i} Q_{1}$ ) would be given not by (31), which suggests that the "tachyon" would be massless in $D=2$, but rather by $-\bar{p}^{a} p_{a}$, which would give (mass) ${ }^{2}=-\frac{15}{8}$ for all $D$. Since for the tachyon one does not have gauge invariance as a guide, nor for $D \leq 24$ can one compactify to a Minkowski spacetime with unbroken Lorentz symmetry, there is an inherent ambiguity in the definition of the tachyon's mass. It should be noted that our preference for $-\bar{p}^{a} p_{a}$ differs from the customary choice in some of the recent literature on two-dimensional gravity, where $-\hat{p}^{a} \hat{p}_{a}$ is taken to be the (mass) $)^{2}$ operator for the tachyon, giving the appearance of a "massless tachyon" in $d=2$ spacetime.

\section{Discussion}

In this paper, we have examined the spectrum of physical states for critical $W_{3}$ string theories, and shown how the intrinsically "non-stringy" coordinate $\varphi_{1}$ appearing in the matter realisations $(1 a, b)$ of the $W_{3}$ algebra is effectively "frozen" at the quantum level by the $W$ constraint, so that the momentum in the $\varphi_{1}$ direction takes the fixed imaginary value $\mathrm{i} Q_{1}$. Moreover, physical states cannot contain any creation operators in the $\varphi_{1}$ direction. The remaining $\left(L_{n}\right)$ constraints on physical states give rise to a spectrum that is rather similar to that for a critical string propagating in $d \neq 26$ dimensions with a background 
charge. The main difference is that there appear to be no massless states in the $W_{3}$ string spectrum.

In deriving our results for the freezing of the $\varphi_{1}$ coordinate, we made the assumption that $\varphi_{1}$ is a spacelike dimension. One might wonder whether one could instead choose to take $\varphi_{1}$ to be timelike, expecting that it will be frozen by the $W$ constraint in any case. A consequence of this choice is that the momentum shift for $p_{1}$, which follows from the analogue of the hermiticity requirement (8), becomes real rather than imaginary. Because of this, the polynomial equations for $p_{1}$ that follow from imposing the $W$ constraint on physical states admit more solutions. For example, at level 0 , the $W$ constraint now implies that $p_{1}$ may be any root of the cubic equation $\left(p_{1}+Q_{1}\right)\left(p_{1}+\frac{6}{7} Q_{1}\right)\left(p_{1}+\frac{8}{7} Q_{1}\right)=0[1,8]$. One also finds in that case that the discussion of section 2 on the absence of $\alpha_{-n}^{1}$ creation operators in physical states is modified. Since for timelike $\varphi_{1}$ the $p^{1}$ momentum shift is real, the characteristic equations for the $W_{0}$ constraint now do allow compatible solutions. For example, at level 1 there are now states compatible with the $W_{0}$ constraint that involve the $\alpha_{-1}^{1}$ oscillator. However, when one calculates the norms of these states, one finds that some of them are negative. Thus even though it is "frozen" by the $W$ constraint, a timelike $\varphi_{1}$ would give rise to a non-unitary theory.

\section{ACKNOWLEDGMENTS}

We are grateful to E. Bergshoeff for discussions. C.N.P. and K.S.S. wish to thank the International Center for Theoretical Physics for hospitality.

\section{REFERENCES}

[1] C.N. Pope, L.J. Romans and K.S. Stelle, "On $W_{3}$ strings," preprint CERN-TH.6171/91, Phys. Lett. B (in press).

[2] C.N. Pope, L.J. Romans and K.S. Stelle, "Anomaly-free $W_{3}$ gravity and critical $W_{3}$ strings," preprint CERN-TH.6171/91, Phys. Lett. B (in press).

[3] J. Thierry-Mieg, Phys. Lett. 197B (1987) 368.

[4] V.A. Fateev and A. Zamolodchikov, Nucl. Phys. B280 (1987) 644;

V.A. Fateev and S. Lukyanov, Int. J. Mod. Phys. A3 (1988) 507.

[5] L.J. Romans, Nucl. Phys. B352 (1991) 829.

[6] I. Antoniadis, C. Bachas, J. Ellis and D.V. Nanopoulos, Nucl. Phys. B328 (1989) 117.

[7] A. Bilal and J.L. Gervais, Nucl. Phys. B326 (1989) 222.

[8] S.R. Das, A. Dhar and S.K. Rama, "Physical properties of $W$ gravities and $W$ strings," preprint, TIFR/TH/91-11;

"Physical states and scaling properties of $W$ gravities and $W$ strings," preprint, TIFR/TH/91-20. 